Journal Club

Editor's Note: These short, critical reviews of recent papers in the Journal, written exclusively by graduate students or postdoctoral fellows, are intended to summarize the important findings of the paper and provide additional insight and commentary. For more information on the format and purpose of the Journal Club, please see http://www.jneurosci.org/misc/ifa_features.shtml.

\title{
Target Enhancement and Distractor Suppression in Naturalistic Visual Search
}

\author{
Tiffany C. Ho and Edward F. Ester \\ Department of Psychology, University of California San Diego, La Jolla, California, 92093-0901 \\ Review of Seidl et al.
}

The human visual system has a limited processing capacity. Consequently, mechanisms of selective attention are required to prioritize behaviorally relevant sensory input. Leading theoretical models propose that selective attention facilitates visual processing by enhancing the cortical representations of behaviorally relevant stimuli (targets) while suppressing the representations of irrelevant stimuli (distractors). While there is ample experimental evidence suggesting that selective attention enhances target representations, evidence for concurrent distractor suppression is mixed (O'Craven et al., 1999; Gazzaley et al., 2005; Peelen et al., 2009).

Typically, studies examining target enhancement and distractor suppression compare cortical responses evoked by attended stimuli to ignored stimuli (e.g., O'Craven et al., 1999; Peelen et al., 2009) or to a passive viewing condition (Gazzaley et al., 2005). In the study by Gazzaley and colleagues (2005), human observers were presented with streams of face and house images and were asked to attend and remember the images from one category (e.g., faces) while ignoring images from the other (e.g., houses). Using functional magnetic resonance imaging (fMRI), these authors examined changes

Received Sept. 19, 2012; revised 0ct. 2, 2012; accepted 0ct. 3, 2012. E.F.E. is supported by NIH T32 MH020002.

Both authors contributed equally to this work.

Correspondence should be addressed to Tiffany C. Ho at the above address. E-mail: tiffnie@gmail.com, eester@ucsd.edu.

DOI:10.1523/JNEUROSCI.4449-12.2012

Copyright $\odot 2012$ the authors $\quad 0270-6474 / 12 / 3216539-02 \$ 15.00 / 0$ in the amplitude of the blood oxygenation level-dependent (BOLD) response in cortical modules that selectively respond to face and house images (the fusiform face area and parahippocampal place area, respectively).

The results of the study by Gazzaley et al. (2005) showed that directing attention to a specific image category was associated with a greater BOLD response in the cortical module selective for that category (relative to a passive viewing condition in which selective attention and memory storage were not required). Conversely, BOLD responses in the cortical module selective for processing the irrelevant category were lower (again relative to the passive viewing condition). These findings are consistent with the hypothesis that attention enhances the processing of targets while suppressing the processing of distractors. However, one limitation of this study (and others like it, such as O'Craven et al., 1999; Peelen et al., 2009) is that the baseline condition used to quantify target enhancement and distractor suppression is inadequate. Specifically, although subjects were never required to attend or remember any of the images in the passive viewing condition, there was nothing to discourage them from doing so. Moreover, if subjects did attend to the images in this condition, then (1) the magnitude of target enhancement and distractor suppression might be underestimated or overestimated, respectively, or (2) enhancement and suppression might be wholly obscured.
In a recent issue of The Journal of $\mathrm{Neu}$ roscience, Seidl et al. (2012) revisited the question of whether selective attention manifests as a mixture of target enhancement and distractor suppression. In the first of two tasks (category discrimination), participants were shown natural scene images containing exemplars from 0-3 relevant categories (cars, people, and trees). Participants were asked to report whether each image contained an exemplar from a target category (e.g., cars). The same image could also contain exemplars from a distractor category (which was always the target category during the previous task run; e.g., trees) or a neutral category (e.g., people). Critically, the neutral category was unknown to the participant and wholly task irrelevant. Thus, responses to this category provide an appropriate baseline for quantifying and comparing target enhancement and distractor suppression.

In the second task (category identification), participants were shown isolated exemplars from the target, neutral, and distractor categories and were asked to detect immediate repetitions of a stimulus exemplar. Next, Seidl et al. (2012) extracted activation patterns occurring in object-selective cortex (OSC; identified with an independent functional localizer) and correlated them with activation patterns for target, distractor, and neutral categories obtained in the category identification task. The logic of this approach is as follows: if exemplars from the target category are processed to a greater extent 
than those from the neutral category in the discrimination task, then the former will dominate the multivoxel responses. Consequently, these responses should be more strongly correlated with targetevoked patterns relative to neutral-evoked patterns in the category identification task. Additionally, if the exemplars from the distractor category are processed to a lesser extent than exemplars from the neutral category, then correlations between multivoxel responses in the discrimination and identification tasks should be lower for distractors relative to the neutral exemplars. Alternatively, if distractor and neutral exemplars are processed to the same extent, then no differences in correlation strength for the neutral and distractor categories should be observed.

Seidl et al. (2012) found that multivoxel responses to scenes containing one or more exemplars from the target category were more strongly correlated with responses evoked by members of the target category than members of the neutral category in the identification task. Conversely, multivoxel responses to scenes containing one or more exemplars from the distractor category were correlated less strongly with multivoxel responses evoked by exemplars from the distractor category than the neutral category in the identification task. Virtually identical findings were obtained when the authors examined responses to scenes containing multiple categories (e.g., target-plus-neutral, target-plus-distractor, target-plus-neutral-plus-distractor, etc) in the recognition task. These findings provide strong evidence in support of the hypothesis that selective attention enhances the cortical representations of stimuli relevant to current behavioral goals while also suppressing cortical representations of irrelevant stimuli.

Before considering the broader implications of this study, a brief methodological point is in order. Generally, one cannot draw firm conclusions about how the representation (or processing) of a stimulus has changed solely by examining changes in multivariate metrics such as correlation or classification accuracy (Serences and Saproo, 2012). Specifically, the core finding reported by Seidl et al. (2012) (i.e., that distractor categories are suppressed relative to neutral categories) could also be explained by an increase in the variability of the multivoxel responses to distractors. For example, on some trials the neutral and distractor categories might be processed to the same extent (i.e., no suppression). On the remaining trials, the distractor category might be processed to a greater extent than the neutral category (e.g., due to a failure to ignore what was once relevant). This variability would weaken the correlation between distractor category responses observed in the discrimination and identification tasks and thereby create the appearance of suppression. Fortunately, Seidl et al. (2012) report behavioral evidence that contradicts this interpretation. Specifically, the authors observed a robust negative relationship between the "strength" of suppression in OSC (defined as the difference in intertask correlations for distractor and neutral categories) and the amount of behavioral interference that a participant experienced (defined as the difference in response times for neutral-only and distractor-only trials of the category discrimination task). This analysis suggests that the effects reported by Seidl et al. (2012) reflect a suppression of distractor information rather than an increase in response variability, and underscore the importance of examining secondary measures when interpreting the results of multivariate $\mathrm{fMRI}$ analyses.

Seidl et al. (2012) propose that target enhancement and distractor suppression occur during visual search (almost) simultaneously. However, one lingering question concerns whether directing attention to exemplars from one category automatically entails a suppression of exemplars from other, irrelevant categories. For example, it is well documented that visual search is slowed when displays contain distractors that were targets on previous trials (Shiffrin and Schenider, 1997; Dombrowe et al., 2011). The magnitude of this effect is relatively small when target and distractor values change on every trial (e.g., Dombrowe et al., 2011), but it increases drastically when the same information is attended for many trials (e.g., Shiffrin \& Schneider, 1977). The experiment by Seidl et al. (2012) was designed such that the target category on a given block was the distractor category in the following block. In this case, distractor suppression might be needed to minimize or eliminate interference generated by the (previously task-relevant) distractors. It would be interesting to know whether distractor suppression also manifests after participants spend just a few trials attending items from one category before they become distractors. Alternatively, distractor suppression might not be all that use- ful in the absence of strong interference. In fact, this may explain why some studies have failed to find any evidence for distractor suppression in OSC; in many of these instances, task demands were mild and the amount of interference generated by irrelevant stimuli was probably quite low (e.g., O'Craven et al., 1999).

To conclude, Seidl et al. (2012) provide novel evidence suggesting that selective attention entails both target enhancement and distractor suppression. However, there remains some ambiguity about whether suppression occurs under all circumstances when attention is deployed. Moreover, the exact relationship between neural measures of attentional enhancement/suppression and behavioral measures of performance has yet to be clarified. For example, does a failure to suppress distractors impair performance with the same magnitude as a failure to enhance targets? Because the present study did not detect evidence of behavioral interference from distractors (i.e., response times and accuracy rates were not significantly different between the distractor and neutral conditions), further work with tasks placing greater attentional demands on participants may be necessary to examine these possibilities in detail.

\section{References}

Dombrowe I, Donk M, Olivers CN (2011) The costs of switching attentional sets. Atten Percep Psychophys 73:2481-2488. CrossRef Medline

Gazzaley A, Cooney JW, McEvoy K, Knight RT, D’Esposito M (2005) Top-down enhancement and suppression of the magnitude and speed of neural activity. J Cogn Neurosci 17: 507-517. CrossRef Medline

O'Craven KM, Downing PE, Kanwisher N (1999) fMRI evidence for objects as the units of attentional selection. Nature 401:584-587. CrossRef Medline

Peelen MV, Kastner S (2009) Neural mechanisms of rapid natural scene categorization in human visual cortex. Nature 460:94-97. CrossRef

Seidl KN, Peelen MV, Kastner S (2012) Neural evidence for distracter suppression during visual search in real-world scenes. J Neurosci 32:11812-11819. CrossRef Medline

Serences JT, Saproo S (2012) Computational advances towards linking BOLD and behavior. Neuropsychologia 50:435-446. CrossRef Medline

Shiffrin RM, Schneider W (1977) Controlled and automatic human information processing, II. Perceptual learning, automatic attending, and a general theory. Psych Rev 84: 127-190. CrossRef 